ACCEPTED MANUSCRIPT

\title{
A computational study of the effects of DC electric fields on non- premixed counterflow methane-air flames
}

To cite this article before publication: Memdouh Belhi et al 2017 J. Phys. D: Appl. Phys. in press https://doi.org/10.1088/1361-6463/aa94bb

\section{Manuscript version: Accepted Manuscript}

Accepted Manuscript is "the version of the article accepted for publication including all changes made as a result of the peer review process, and which may also include the addition to the article by IOP Publishing of a header, an article ID, a cover sheet and/or an 'Accepted

Manuscript' watermark, but excluding any other editing, typesetting or other changes made by IOP Publishing and/or its licensors"

This Accepted Manuscript is @ 2017 IOP Publishing Ltd.

During the embargo period (the 12 month period from the publication of the Version of Record of this article), the Accepted Manuscript is fully protected by copyright and cannot be reused or reposted elsewhere.

As the Version of Record of this article is going to be / has been published on a subscription basis, this Accepted Manuscript is available for reuse under a CC BY-NC-ND 3.0 licence after the 12 month embargo period.

After the embargo period, everyone is permitted to use copy and redistribute this article for non-commercial purposes only, provided that they adhere to all the terms of the licence https://creativecommons.org/licences/by-nc-nd/3.0

Although reasonable endeavours have been taken to obtain all necessary permissions from third parties to include their copyrighted content within this article, their full citation and copyright line may not be present in this Accepted Manuscript version. Before using any content from this article, please refer to the Version of Record on IOPscience once published for full citation and copyright details, as permissions will likely be required. All third party content is fully copyright protected, unless specifically stated otherwise in the figure caption in the Version of Record.

View the article online for updates and enhancements. 


\title{
A computational study of the effects of DC electric fields on non-premixed counterflow methane-air flames
}

\section{Memdouh Belhi ${ }^{\mathrm{a}}$, Bok Jik Lee ${ }^{\mathrm{b}}$, Fabrizio Bisetti ${ }^{\mathrm{c}}$,} Hong G. Im*,a

${ }^{a}$ King Abdullah University of Science and Technology, Clean Combustion Research Center, Thuwal 23955, Saudi Arabia

${ }^{\mathrm{b}}$ Department of Mechanical Engineering, Gwangju Institute of Science and Technology, Gwangju, South Korea ${ }^{c}$ Department of Aerospace Engineering and Engineering Mechanics, University of Texas at Austin, Austin, TX 78712-1085, USA

E-mail: hong.im@kaust.edu.sa

July 2017

\begin{abstract}
.
Two-dimensional axisymmetric simulations for counterflow nonpremixed methaneair flames were undertaken as an attempt to reproduce the experimentally observed electro-hydrodynamic effect, also known as the ionic wind effect, on flames. Incompressible fluid dynamic solver was implemented with a skeletal chemical kinetic mechanism and transport property evaluations. The simulation successfully reproduced the key characteristics of the flames subjected to DC bias voltages at different intensity and polarity. Most notably, the simulation predicted the flame positions and showed good qualitative agreement with experimental data for the current-voltage curve. The flame response to the electric field with positive and negative polarity exhibited qualitatively different characteristics. In the negative polarity of the configuration considered, a non-monotonic variation of the current with the voltage was observed along with the existence of an unstable regime at an intermediate voltage level. With positive polarity, a typical monotonic current-voltage curve was obtained. This behavior was attributed to the asymmetry in the distribution of the positive and negative ions resulting from ionization processes. The present study demonstrated that the mathematical and computational models for the ion chemistry, transport, and fluid dynamics were able to describe the key processes responsible for the flame-electric field interaction.
\end{abstract}

Keywords: counterflow flame, DC electric field, electro-hydrodynamic force, positive and negativê charges

Submitted to: J. Phys. D: Appl. Phys. Special Issue on Multiscale Modeling of NonEquilibrium Plasma Discharges 


\section{Introduction}

High temperature reaction zones in flames not only generate intermediate-radicals, but also a finite level of electrically charged species by the inherent chemi-ionization processes [1]. These charged molecules may impart additional effects on the aerodynamic and chemical characteristics of the flames in the presence of an external electric field. As such, for decades there have been attempts to utilize an imposed electric field to improve combustion performance in practical devices $[2,3]$. Applications include the enhancement of flame stabilization in laminar and turbulent burners $[4,5,6,7,8,9]$ and the reduction of soot formation $[10,11,12]$. While these studies provided evidence that electric fields do affect flames and combustion, the detailed underlying physical and chemical mechanisms are not understood clearly.

One of the most plausible explanations is the electro-hydrodynamic effect, also called the ionic wind effect, which refers to the generation of a body force due to the collision of charged particles with the surrounding neutral molecules, altering the convective transport in the bulk gases. Many studies reported the effects of the electrohydrodynamic forces on increasing the burning velocity [13, 14], on modifying the characteristics of the laminar bunsen flames $[9,15]$, and on extending the stabilization limits of the nonpremixed jet flames subjected to an electric field oriented in the streamwise direction $[5,16,17]$. The subject has been extensively discussed by Lawton and Weinberg [2] and Fialkov [3], providing qualitative physical understanding of the process. However, further investigations are needed in order to understand the effect of the polarity of the applied electric field and the roles played by different types of charged species $[18,19]$. Moreover, the effects brought by the electro-hydrodynamic force are most likely specific to the configuration, depending on the placement of the electrodes, flame location and orientation with respect to the electric field and flow rates. It is generally understood that ionic wind blows only in the same direction of the positive ions, i.e. toward the lower potential electrode (cathode) [20, 21]. However, other recent studies $[19,22]$ have reported that, under certain mixture and electric field conditions, ionic wind may be directed to both cathode and anode directions. To understand these complex interactions, high fidelity numerical models are needed to predict the behavior quantitatively.

Numerical simulations of flames in response to electro-hydrodynamic forces have been studied in the past decades $[17,18,23,24]$. The determination of the induced electro-hydrodynamic force requires information on the spatial distribution of electrons and ions. Therefore, the model must accurately predict both the production rates and transport coefficients of all relevant charged species. Validation of models is challenging as the quantitative measurements of ion concentrations are difficult and reliable quantitative experimental data is scarce, thus leading to uncertainties in the simulation results and their interpretations.

Despite the many challenges, significant progress has been made recently through the improvements in the ionization kinetics in methane flames $[25,26,27,28]$ and 
molecular transport [29]. The authors' group have also contributed with an improved chemical kinetic mechanism [28] and transport properties [30, 31], demonstrating the ability to predict the current-voltage curves in premixed burner-stabilized flames $[25,26]$. Based on the advanced chemical description of key chemi-ionization processes, the present study undertakes two-dimensional simulations of non-premixed counterflow laminar methane-air flames under a DC electric field in order to reproduce the observations made in a recent experimental study [19], where measurements of the flame positions and current densities are available for various applied voltages, allowing a systematic validation of the model predictions. Simulations with AC electric fields were not considered in the present work since no experimental data for the same configuration is available. While the counterflow flame configuration has been commonly analyzed with an one-dimensional formulation [32], the complex coupling between the electric fields and chemi-ionization processes suggested that a more realistic multi-dimensional description is necessary.

In the following, the mathematical formulations and model description for the ion chemistry and transport are presented. Next, problem configuration with basic parametric conditions along with the numerical procedure are described. Comparisons between numerical simulation results and experimental data for the flame positions and current-voltage curve are then shown. Finally, a detailed analysis of the spatial distribution of charges, electro-hydrodynamic force and flow velocity is presented to explain the observed behavior of the flame under a bias voltage. Conclusions are discussed in Section 6. The reaction mechanism is provided as Supplementary Material.

\section{Physical model}

\subsection{Governing equations}

The equations describing the action of an electric field on a weakly ionized gas, such as a flame, are different in the thermal and non-thermal regimes. As a criterion to distinguish those two regimes, the reduced electric field $E / N$ is often used, where $N$ is the number density of the gas mixture and $E$ is the electric field strength. A previous analysis of non-thermal charges in methane-air flames suggests that electrons and ions are in thermal equilibrium with the background neutral combustion gases for reduced electric field strengths up to $140 \mathrm{Td}[33,34]$. Since $E / N$ is much lower than this threshold in the configurations considered in the present work $(E / N \approx 10 \mathrm{Td}[19])$, charges are assumed to remain in the thermal regime.

Modeling of a flame subjected to a weak bias voltage is governed by the conservation equations for reacting flows in the presence of electro-hydrodynamic forces. This involves the conservation of mass, momentum, total energy and species densities, respectively, written as [35]:

$$
\frac{\partial \rho}{\partial t}+\nabla \cdot(\rho \mathbf{U})=0
$$




$$
\frac{\partial \rho \mathbf{U}}{\partial t}+\nabla \cdot(\rho \mathbf{U} \otimes \mathbf{U}+p \mathbf{I}-\tau)=\rho \sum_{k}^{N} Y_{k} \mathbf{f}_{k},
$$$$
\frac{\partial \rho E}{\partial t}+\nabla \cdot[\rho \mathbf{U} E+\mathbf{q}-(\tau-p \mathbf{I}) \cdot \mathbf{U}]=-\sum_{k}^{N} h_{k} \dot{\omega}_{k}+\rho \sum_{k}^{N} Y_{k} \mathbf{f}_{k}\left(\mathbf{U}+\mathbf{V}_{k}\right),(3)
$$$$
\frac{\partial \rho Y_{k}}{\partial t}+\nabla \cdot\left[\rho\left(\mathbf{U}-\mathbf{V}_{k}\right) Y_{k}\right]=W_{k} \dot{\omega}_{k} .
$$

The above set of equations solve for the density $\rho$, the velocity vector $\mathbf{U}$, the total energy $E$ and the mass fractions $Y_{k}$ of $N$ reacting species (both neutral and charged). $t$ is time, $p$ is pressure calculated by the equation of state and $\mathbf{q}$ is the heat flux calculated from Fourier's law. $\tau$ and $\mathbf{I}$ are the viscous stress and identity tensors, respectively. $W_{k}, \mathbf{V}_{k}$, $\dot{\omega}_{k}$ and $h_{k}$ are the molar mass, the diffusion velocity, the molar source term, and the enthalpy of formation of species $k$, respectively. $f_{k}$ is the volume force acting on species $k$.

Under the assumption that the density gradients in the charged particle density are negligible, the electro-hydrodynamic force per unit volume is written as $[2,36,37]$ :

$$
\mathbf{F}=\sum_{k}^{N} q_{k} e \mathbf{E} n_{k},
$$

where $q_{k}$ and $n_{k}$ are the charge number and the number density of charged species $k$, respectively. $e=1.602 \times 10^{19} \mathrm{C}$ is the elementary charge and $\mathbf{E}$ is the electric field vector defined by the Gauss law as:

$$
\nabla \cdot \mathbf{E}=-\nabla^{2} V=\frac{\sum_{k} q_{k} e n_{k}}{\epsilon_{0}}
$$

where $V$ is the electric potential and $\epsilon_{0}=8.854 \times 10^{-12} \mathrm{~F} \cdot \mathrm{m}^{-1}$ is the vacuum permittivity. The electric force term appears in the equations of momentum and energy, but its contributions in the total energy conservation equation was found to be negligible $[17,18]$.

The species diffusion velocity $\mathbf{V}_{k}$ depends on the charge of the particles. For neutral species, the diffusion velocity was expressed by Fick's law [35, 38]. For charged species, an additional mass flux, namely the drift flux due to the electric mobility, $\mu_{k}$, is included:

$$
\rho Y_{k} \mathbf{V}_{k}=-\rho D_{k} \frac{W_{k}}{W} \nabla X_{k}+q_{k} \rho Y_{k} \mu_{k} \mathbf{E}
$$

where $X_{k}$ and $D_{k}$ are the mole fraction and the diffusion coefficient of species $k$, respectively. $W$ is the mixture molar mass.

\subsection{Transport properties}

The diffusion coefficients of neutral species were approximated by using the unity Lewis number assumption, so that the diffusion coefficient $D_{k}$ is set equal to the thermal diffusion coefficient $D_{t h}=\lambda / \rho c_{p}$ where $\lambda$ is the thermal conductivity and $c_{p}$ is the specific heat of the mixture [35]. This approximation was validated by comparing to 
detailed one-dimensional simulations with detailed transport properties. The use of the unity Lewis number assumption for the ion transport properties may degrade the accuracy of the calculations of the electro-hydrodynamic force, because it does not take into account the long range potential induced by the charge of ions [30]. Therefore, the ion mobilities and diffusivities were determined using the mixture-average rules with interaction potentials, which are appropriate for collisions involving charged species.

The $(\mathrm{n}, 6,4)[39]$ and Coulomb [40] interaction potentials were used to describe ionneutral and ion-charge interactions, respectively. The details of the calculation method of ion transport coefficients are provided in Refs. [30,34]. The ion transport model was found to reproduce the current-voltage curves reasonably well in flat burner-stabilized flames [28, 41].

Electron transport coefficients were computed as a function of temperature and mixture composition by adopting the methodology by Bisetti and El-Morsli [31]. The method uses cross-section data for collisions between electrons and the most abundant neutral species in a reactive mixture and combustion products. The cross-sections were taken from the LXcat repository [42]. The variation of mobility with the reduced electric field was not taken into account since the electrons are thermal.

\subsection{Chemical kinetic mechanism}

In Ref. [28], a detailed reaction mechanism involving 326 species and 1898 reactions to describe the chemistry of ions in flames was developed and validated. The model coupled the detailed neutral AramcoMech 1.4 mechanism [43, 44], which was optimized to improve the prediction of the $\mathrm{CH}$ concentration, with an updated ion chemistry mechanism [29]. Rate parameters of ion reactions and thermodynamic data of charged species have been updated based on the most recent data $[45,46]$. The optimization of $\mathrm{CH}$ chemistry was motivated by the important role of $\mathrm{CH}$ in the production of charges in flames through chemi-ionization reactions $[1,28,47]$. The model has been demonstrated to yield good quantitative agreement with experimental data for the electric current measured from atmospheric-pressure, premixed, burner-stabilized methane-air flames [26].

However, integrating such a detailed model into multi-dimensional numerical simulations incurs a significant computational cost, especially considering that the electron drift velocity $\mathbf{v}_{d}^{e}=\mu^{e} \mathbf{E}$ demands an extremely small time step [18]. For the present study, a simplified approach was adopted to combine a skeletal mechanism capable of predicting major neutral species and the fundamental properties of combustion in methane-air flames with the main ionization mechanism in the detailed model in Ref. [28]. A skeletal mechanism consists of far fewer species than a detailed mechanism, leading to significant savings in terms of memory and computational time. Only ions and reactions most important for the quantitative prediction of the electric currents were retained. The $\mathrm{H}_{3} \mathrm{O}^{+}$ion was considered as the sole carrier of positive chárge, while the negative charge was represented by electrons and 5 negative ions 
$\left(\mathrm{O}_{2}^{-}, \mathrm{O}^{-}, \mathrm{OH}^{-}, \mathrm{CHO}_{3}^{-}\right.$and $\left.\mathrm{CO}_{3}^{-}\right)$. The proposed strategy led to a significantly reduced reaction mechanism with 25 species and 76 reactions, without loss of fidelity that can be obtained with the detailed model in Ref. [28]. The detailed description of the simplified chemistry model along with its validation against the detailed model are given in the Supplementary Material.

\subsection{Boundary conditions of charged species at electrodes}

The boundary conditions proposed in Ref. [17] were adopted for the charged species. The treatment of the charged species at the electrodes assumes that the drift flux of positive ions is directed toward the cathode (negative pole) and is zero at the anode (positive pole), while the drift fluxes of electrons and negative ions are zero at the cathode and are directed toward the anode [48]. Based on these principles, the mass fractions of negative (positive) charges are set to zero at the cathode (anode) and their gradients are set to zero at the anode (cathode).

\section{Configuration and numerical procedure}

The experimental configuration in Ref. [19] consisted of a non-premixed counterflow flame between two nozzles. It was modeled by a two-dimensional axisymmetric computational domain as shown in Fig. 1. Near the central axis, methane-nitrogen and oxygen-nitrogen mixtures were supplied from the bottom and top nozzles at a diameter of $1 \mathrm{~cm}$, respectively, creating a nearly planar nonpremixed flame between the two nozzles. The opposed nozzles were surrounded by co-flowing nitrogen jets through the outer nozzles with a diameter of $2 \mathrm{~cm}$, in order to isolate the flame from the ambient air. Both the core stream of reactants and the inert coflow were at the velocity of $20 \mathrm{~cm} / \mathrm{s}$, temperature of $300 \mathrm{~K}$, and atmospheric pressure. The distance between two opposing

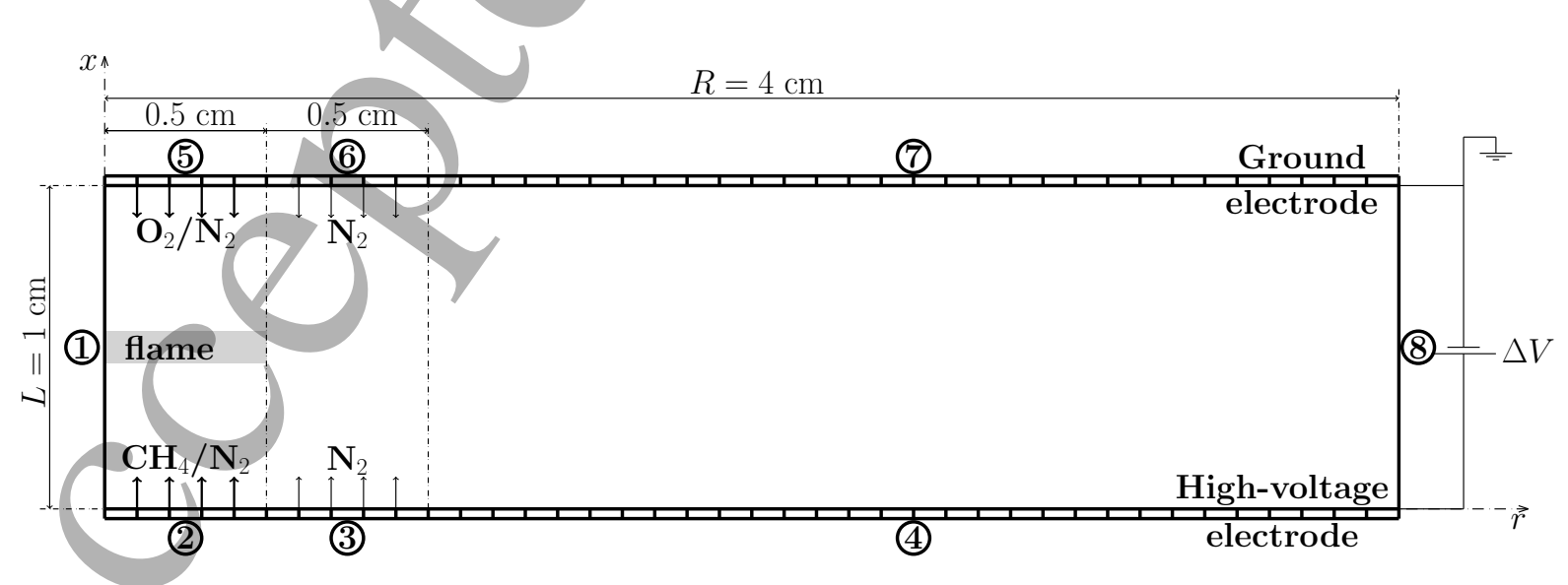

Figure 1. Schematic of the computational geometry inspired by the experimental setup in Ref. [19]. Bold labels inside circles (numbers from 1 to 8 ) correspond to the problem's boundary conditions listed in table $1 . x$ and $r$ represent axial and radial coordinates, respectively. 
nozzles, $L$, was $1 \mathrm{~cm}$. Two mesh electrodes, each at $8 \mathrm{~cm}$ in diameter were located at the nozzle exits. The electrodes were oriented so that their surface is normal to the nozzle axis. The electric field was established by applying a positive or negative DC voltage difference $\Delta V_{0}$ to the lower electrode, while the upper one was grounded. While various fuels were tested in Ref. [19], for the present numerical simulations only the cases with methane fuel were considered as the employed reaction mechanism involves the ion chemistry validated for methane flames [29, 49, 50, 51].

Modeling of a non-premixed flame between two opposing nozzles in an axisymmetric flow is generally reduced to a one-dimensional problem $[32,52]$. However, the standard one-dimensional formulation cannot include an axial body force since the axial velocity is obtained from the radial momentum equation. Therefore, to obtain ionic wind in simulations, it was considered that at least a two-dimensional domain is necessary. For computational cost consideration, a two-dimensional geometry was found to be adequate to reproduce the key experimental observations. As such, the/present model rules out predicting more complex three-dimensional effects associated with the flow entrainment, as observed in some experimental studies, [21, 22]. Note that, as the radial extent of the electrodes in the configuration studied here is sufficiently large compared to the separation distance (see Fig. 1), the drift fluxes in the axial direction and the associated electro-hydrodynamic forces are much more significant than those induced in the radial direction.

The configuration shown in Fig. 1 was simulated by using the reactingFoam solver of the OpenFOAM software [53] modified for the task. Several modifications were implemented in order to describe the electric field effects. Most notably, the electric force (Eq. 5) was added to the momentum equation and the mass flux in the transport equations for charged species was modified to include the drift diffusion flux. The electric potential and strength were added as additional variables and their distributions were determined by the Gauss law. The methodology to compute the ion and electron transport properties was also implemented. The solver files are uploaded as Supplementary material. The set of Navier-Stokes equations (Eqs. 1 to 3) along with species transport equations (Eq. 4) and the Poisson equation for the electric potential (Eq. 6) were solved to satisfy the boundary conditions shown in Table 1. Standard linear Gaussian finite volume integration was adopted for the spatial derivatives with first order implicit time integration [54].

The grid dependence of the solutions was assessed by considering two cell sizes, $\delta=50$ and $100 \mu \mathrm{m}$. The two cases of resolution showed negligible differences on the flame characteristics, in terms of the axial position and current density. Therefore, $\delta=100 \mu \mathrm{m}$ was adopted in all subsequent simulations, which resulted in 100 and 400 cells in the axial and radial directions, respectively. The simulations presented in this work were carried out on 384 CPU-cores with time step of 5 ns on average, costing typically $0.1 \mathrm{M}$ core-hours per simulation for a given applied voltage.

The flames under study are characterized by a stoichiometric mixture fraction, $Z_{s t}=1 /\left(1+\nu Y_{F, 0} / Y_{O, 0}\right)=0.5$, where $\nu$ is the stoichiometric ratio and $Y_{F, 0}$ and 


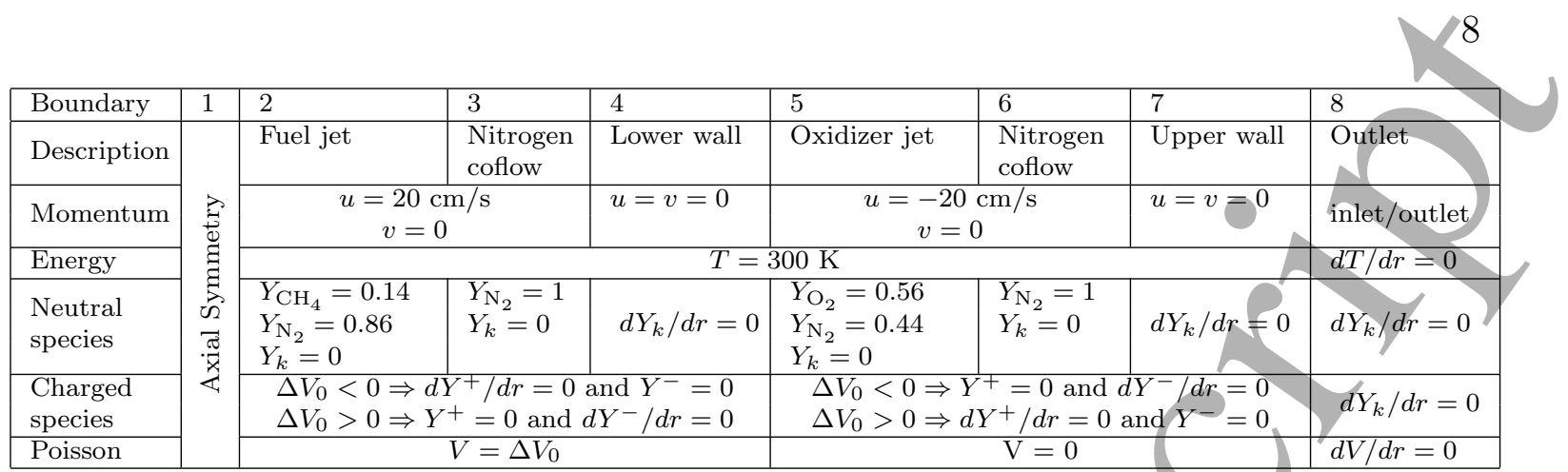

Table 1. Boundary conditions corresponding to the computational domain in Fig. 1. $u$ and $v$ are the radial and axial components of velocity, respectively. The + and subscripts denote positive and negative charged species, respectively.

$Y_{O, 0}$ represent mass fractions of methane and oxygen in the fuel and oxidizer streams, respectively. The mass fractions of methane, oxygen and nitrogen at the boundaries as well as those for the other neutral and charged species are listed in Table 1. With the nitrogen dilution, the flame is positioned in the center near the stagnation plane [55], allowing a low velocity environment near the reaction zone suitable for observing electro-hydrodynamic force effects.

As for the computational procedure, the simulation was first carried out without the application of an electric field. The obtained flame stabilized at a certain location, which is considered the baseline axial position. The flame position is defined as the axial coordinate of peak heat release rate at the centerline. Thereafter, voltages at increasing strength and different polarities were applied, and the resulting flame response was monitored.

Detailed experimental data on flame axial locations and electric current densities for various negative voltages are available in Ref. [19], which were used for comparison. Subsequently, the computational study was further extended to consider positive voltages in order to obtain a more comprehensive picture on the effects of electric fields on flames.

\section{Model evaluation}

\subsection{Flame position behavior}

To assess the fidelity of the model, the flame positions obtained from the present numerical simulations for various negative voltages are compared to measured data in Fig. 2. When the absolute value of the applied potential difference was smaller than a threshold value, no noticeable change in flame position was observed. The voltage bias necessary to produce visible effects on the flame position was around $1 \mathrm{kV}$, which is in good agreement with the experiment. As the potential difference approached $1 \mathrm{kV}$, the flame first moved and settled closer to the anode. Subsequently, as the voltage was increased further, an unstable regime was observed such that the 
Figure 2. Flame axial locations for various negative voltages compared to experimental values [19].

flame fluctuated radially and axially without reaching a steady position (see a movie in the Supplementary Material). A similar oscillatory behavior was also observed at the transition to saturation under certain flow conditions in a coflowing non-premixed methane/air flame [56].

After the end of the unstable regime $\left(\Delta V_{0}=-1.14 \mathrm{kV}\right.$ in the simulation and $\Delta V_{0}=-1.26 \mathrm{kV}$ in the experiment), the flame was relocated towards the cathode. The largest variation in flame position was achieved just after the unstable regime. As the applied voltage was further increased (around $2 \mathrm{kV}$ in both experiments and simulations), the flame was stabilized at $4 \mathrm{~mm}$ from the lower nozzle (cathode side) and levels off at higher voltages.

The prediction of the flame position is in good agreement with the experiment. The quantitative agreement in the sub-saturation regime is less satisfactory, most notably at the end of the unstable regime where a relative difference of $35 \%$ in comparison to the experimental data was observed. As demonstrated in previous studies [26, 27], the amount of the electric current drawn from the flame before saturation depends strongly on the transport properties of charged species. Differences between simulations and experiments may thus be due to uncertainties in the prediction of transport coefficients of charged species. Recall that at saturation, the induced current depends only on the number of ions or electrons produced in the flame via chemi-ionization [36].

Figure 3 shows the flame position behavior with positive voltages applied at the lower nozzle. In contrast to the results with negative polarity, the flame location monotonically moves towards the cathode as the voltage increases, without exhibiting any unstable behavior. The maximum change in flame position was obtained at saturation current point, beyond which the flame position levels off. The maximum flame dísplacement obtained for the positive bias is nearly the same as that obtained for the negative bias. A minimum positive voltage of $0.75 \mathrm{kV}$ was required to observe any effect on the flame position, while the maximum flame displacement (around $5.5 \mathrm{~mm}$ ) 


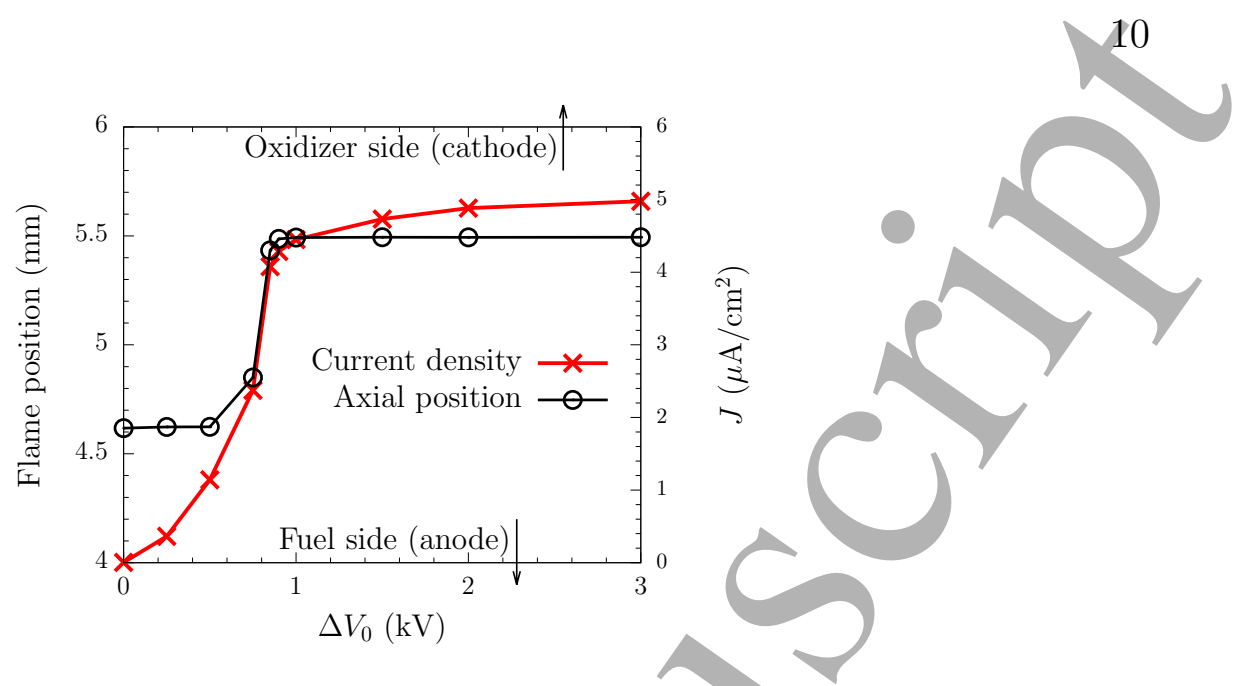

Figure 3. Flame axial locations (black line with circle symbols) and current densities (red line with cross symbols) for various positive bias voltages.

was reached at $\Delta V=0.9 \mathrm{kV}$. Note that model predictions for positive voltages cannot be evaluated because of the lack of experimental data. The current density behavior shown in Fig. 3 will be discussed in detail in the next sub-section.

Figure 4 shows the simulation results of the temperature iso-contours for negative voltages. The flame position was represented by the stoichiometric mixture fraction surface, $Z=Z_{s t}=0.5$, which is valid for low strain rate conditions under study. Note that the flame exists only in the center region $(r<1 \mathrm{~cm})$, as the mixture is not flammable at larger $r$ due to nitrogen dilution and air entrainment. It is seen that the flame shape is nearly flat, except for case (c) which corresponds to the condition near the unstable regime. For all other conditions, the overall shape of the flame and the temperature field are unchanged by the application of the electric field, but they are only shifted toward the lower or upper plane depending on the polarity and intensity of the applied voltage. These observations are consistent with experimental findings [19].

The above results demonstrate that the simulations properly capture the essential physical processes of the electric field effects on the flames.

\subsection{Current densities}

The extent to which an electric field modifies a flame is limited by the saturation current that may be drawn from a flame $[2,27,36]$. Therefore, an accurate prediction of currents is crucial in simulating the response of flames to electric fields adequately.

The current density consists of the contributions from the convective, diffusive and drift fluxes summed over all the charged species:

$$
\mathbf{J}=\sum_{k}^{N} \mathbf{J}_{k}=\sum_{k}^{N} e q_{k} n_{k}\left(\mathbf{U}-\mathbf{V}_{k}\right)
$$

As the fluxes of charged species in the radial direction are insignificant near the centerline, the following relation is obtained as a result of charge conservation by 


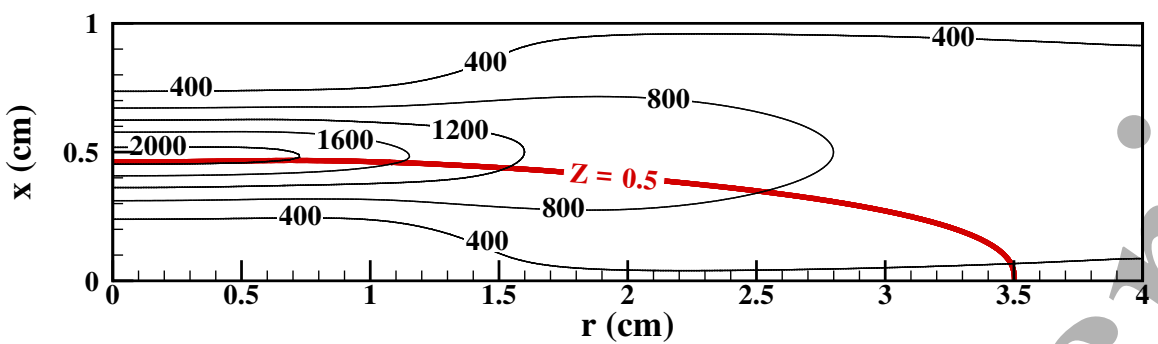

(a) $\Delta V_{0}=0 \mathrm{~V}$

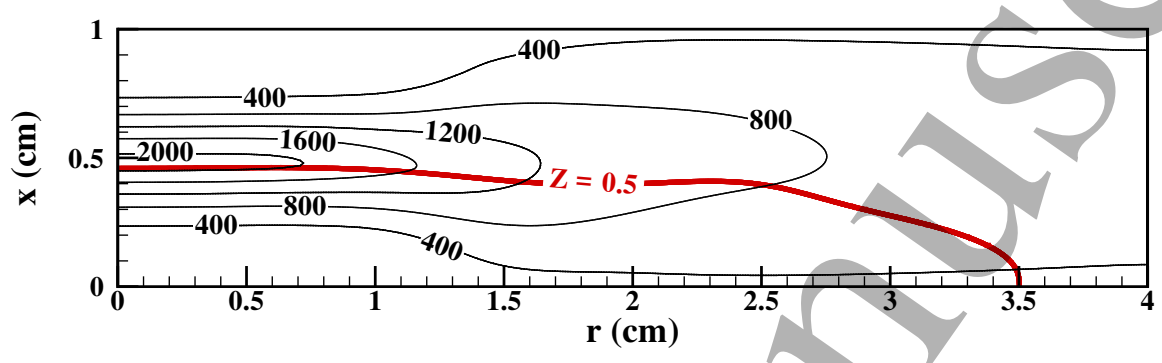

(b) $\Delta V_{0}=-0.5 \mathrm{kV}$

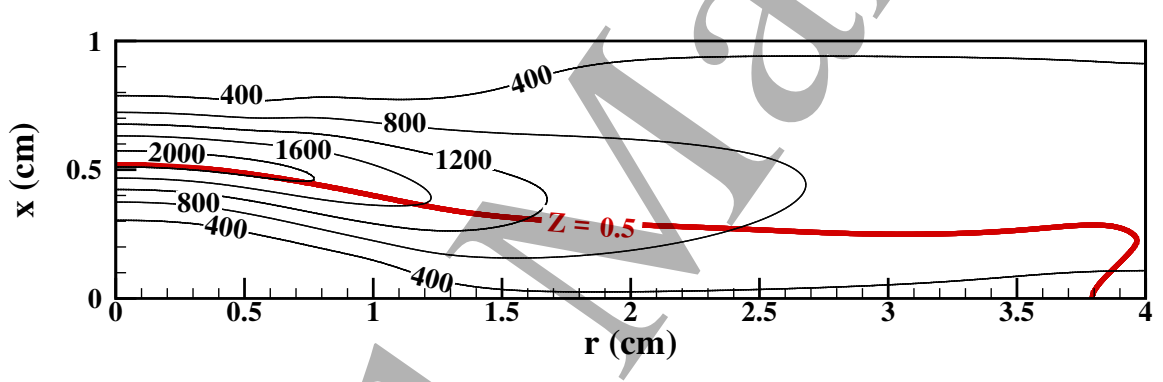

(c) $\Delta V_{0}=-1.05 \mathrm{kV}$

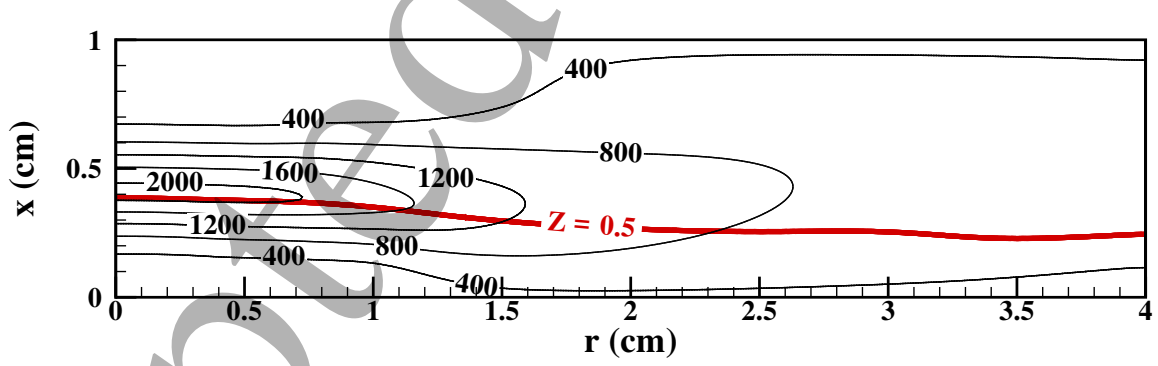

(d) $\Delta V_{0}=-3 \mathrm{kV}$

Figure 4. Iso-contours of temperature with (a) no applied potential, (b) $\Delta V=-0.5$ $\mathrm{kV}$, (c) $\Delta V=-1.05 \mathrm{kV}$ and (d) $\Delta V=-3 \mathrm{kV}$. Thick red line corresponds to $Z=Z_{s t}=0.5$.

summing up the mass fraction transport equations (Eq. 4) over $N$ species:

$$
\frac{\partial J}{\partial x} \approx \sum_{k}^{N} e q_{k} N_{A} \dot{\omega}_{k}=0,
$$

where $x$ denotes the axial direction (see Fig. 1) and $N_{A}$ is the Avogadro number. The net current density drawn from the flame is thus considered nearly uniform in the axial 
direction for a given applied voltage [26, 27].

Current densities obtained from the present numerical simulations for negative bias voltages are compared to experimental values in Fig. 5. The simulations qualitatively reproduce the evolution of the electric current with the applied electric potential. Prior to the unstable regime, the evolution of the current as a function of the applied potential is approximately quadratic with respect to the bias voltage. This behavior is consistent with the previous theoretical study in Ref. [27]. The peak current density was obtained just after the unstable regime, where the largest change in flame position was also observed. When the voltage is increased further, a saturation/condition was reached, i.e. a voltage increase above a certain value has practically no effect on the current density.

Note that the quantitative differences between measured and simulated saturation currents are relatively large (up to $40 \%$ ), compared to those for the flame axial locations (see Fig. 2). Experimentally, the current density was computed by the measured current divided by the projected flame surface area (based on the flame luminosity) onto the horizontal plane [19]. As such, significant uncertainties are expected in the experimental determination of the current densities. Nevertheless, the general trend is properly reproduced by the numerical model.

The maximum current density that can be drawn from the flame is determined by the production rate of charged species, estimated by [2, 26, 27]:

$$
J_{\text {max }} \approx e N_{A} \int_{x=0}^{x=L} \dot{\omega}_{p}^{i} d x
$$

where $\dot{\omega}_{p}^{i}$ is the molar ion production rate. The maximum current density is therefore a function of the reaction of the neutral species participating in the ion production process $\left(\mathrm{CH}_{3}, \mathrm{OH}\right.$ and $\mathrm{O}$ in our case; see the Supplementary Material.). As the concentrations of these neutral species change with the applied voltage as a result of the hydrodynamic

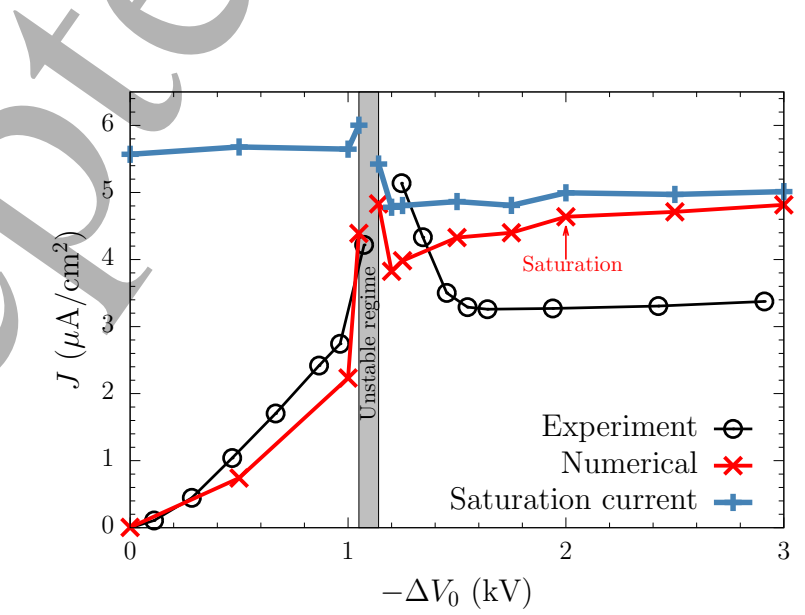

Figure 5. Current densities as a function of the negative bias voltages from numerical prediction and experimental measurements [19]. The maximum current density that can be drawn from the flame (Eq. 10) is shown also. 
conditions experienced by the displaced flames, the maximum current also varies with the applied voltage as shown in Fig. 5 (blue line with plus symbols). In other words, due to modifications to the velocity field, which induces modifications to the spatial distributions of temperature and reactive species, the rate of chemi-ionization changes as well.

As shown in Fig. 5, the gap between the current drawn from the flame and the saturation current decreases with increasing the voltage. As the current reaches saturation, the flame characteristics do not respond to further increases in the bias voltage. The flame behavior is influenced by increasing voltage as long as the induced current is lower than the rate of formation of charged particles.

Results of the current density variation for positive voltages was shown in Fig. 3, in which the behavior is consistent with a typical monotonic curve observed in previous studies $[2,26,27,36]$. For voltages above $0.9 \mathrm{kV}$, the flame position remains constant while the current density continue to rise slightly until around $\Delta V_{0}=2 \mathrm{kV}$ when the saturation condition is reached. The small level of the current density increase did not have a noticeable effect on the velocity field to modify the flame location.

\section{Effects on the flame structure}

To understand more details of the flame behavior in the presence of the electric field, the spatial distributions of charges, electric potential, and axial electric force along the centerline $(r=0)$ are analyzed for three negative voltages $(-1.05,-1.14$ and $-2 \mathrm{kV})$ and two positive voltages $(2$ and $0.85 \mathrm{kV})$. The first negative voltage $(-1.05 \mathrm{kV})$ corresponds to the situation where the flame was repositioned towards the anode before the unstable regime. The second case $(-1.14 \mathrm{kV})$ corresponds to the end of the unstable regime, where a maximum move of the flame towards the cathode was observed. The third and forth cases $(-2$ and $2 \mathrm{kV})$ represent a flame in the saturated regime with negative and positive voltages, respectively. Finally, the fifth case $(0.85 \mathrm{kV})$ corresponds to a flame in the sub-saturated regime with a positive polarity.

\subsection{Distribution of charges}

The distributions of positive ions, electrons and negative ions along the centerline $(r=0)$ are shown in Fig. 6. At the baseline case without an external voltage, the self-sustained electric field of the flame slows down the diffusion of the electrons and accelerates that of ions, resulting in a charge neutrality and a zero net current. This process is referred to as ambipolar diffusion. When an electric field is applied, charged species are driven by the local gradient of the electric potential; the positive charges are transported from the reaction zone towards the cathode and negative charges move in the opposite direction towards the anode.

As shown in Figs. 6(a) and 6(d), positive ions are abundant in the space situated between the flame and cathode for both positive and negative voltages. Figs. 6(b) and 
6(e) show that, as the applied potential difference increases, electrons are removed from the reaction zone and their concentration becomes insignificant. This is due to the high drift flux of electrons caused by their high mobility. The distribution of total number density of negative ions displayed in Fig. 6(f) shows that the overall concentrations of negative ions are negligible when a positive voltage is imposed. However, negative ions are dominant in the space between the flame and anode when a negative voltage is applied (see Fig. 6(c)). In this case, the anode is positioned at the oxidizer side where oxygen molecules abound, so that electrons moved from the reaction zone to the anode are attached to oxygen via three-body reactions to form $\mathrm{O}_{2}^{-}$ion $\left(\mathrm{O}_{2}+\mathrm{e}^{-}+\mathrm{M} \rightleftharpoons\right.$ $\mathrm{O}_{2}^{4}+\mathrm{M}$; see reaction mechanism in the Supplementary Material). This behavior is 


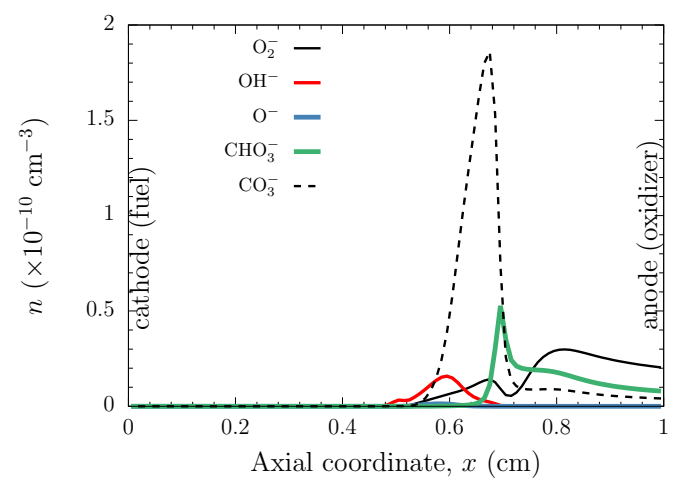

(a) $\Delta V_{0}=-1.05 \mathrm{kV}$

Figure 7. Individual negative ions' densities along the centerline $(r=0)$ with (a) $\Delta V_{0}=-1.05 \mathrm{kV}$ and (b) $\Delta V_{0}=-2 \mathrm{kV}$.

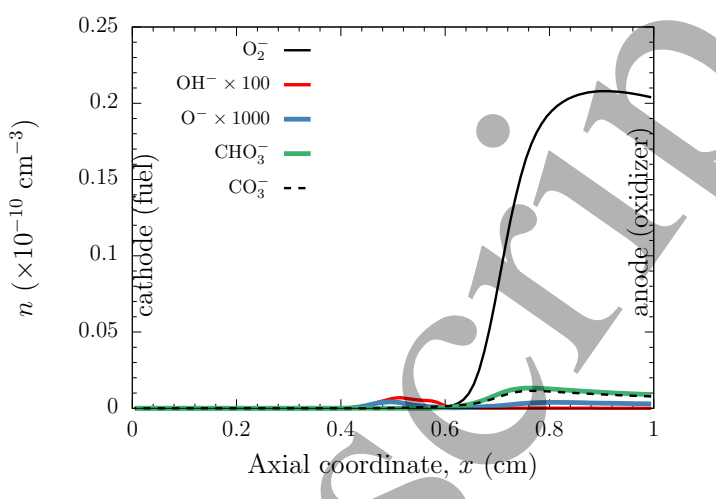

(b) $\Delta V_{0}=-2 \mathrm{kV}$

the centr

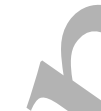

evident in Fig. 7 for two negative voltages, where $\mathrm{O}_{2}^{-}$ion is the most abundant ion in the region near the anode for both voltages.

The distribution of charged species described above leads to the electric potential distribution shown in Fig. 8. At sub-saturation regimes $\left(\Delta V_{0}=-1.05 \mathrm{kV}, \Delta V_{0}=-1.14\right.$ and $\Delta V_{0}=0.85 \mathrm{kV}$ ), the distribution of the electric potential shows three zones: a central plateau region around the flame where electric potential is nearly constant due to charge neutrality, and two regions near the cathode and anode, respectively, where the potential gradients develop. Above the saturation condition $\left(\Delta V_{0}=-2\right.$ and $\left.2 \mathrm{kV}\right)$, the plateau region disappears and the electric potential varies monotonically between the electrodes. These results are consistent with previous observations in burner-stabilized flames [27, 57, 58].

Note that the radial component of the electric field is negligible around the central region of the flame where the flame surface is flat, resulting in the electric force

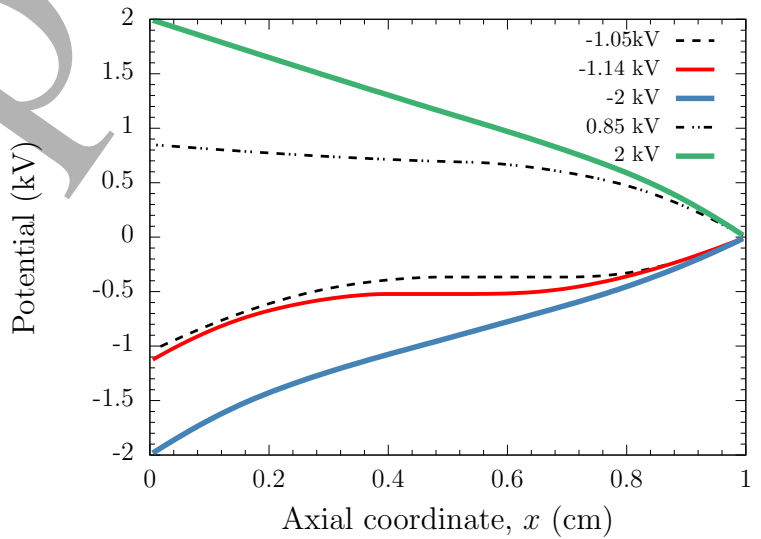

Figure 8. Distribution of electric potential along the centerline $(r=0)$ for various voltages. 
perpendicular to the flame surface. Further away in the radial direction, however, once the flame surface becomes curved, a significant level of the radial component of the electric force may develop, resulting in further bending of the flame towards the cathode as shown in Fig. 4.

\subsection{Electro-hydrodynamic force}

Figure 9 shows the distribution of the axial electro-hydrodynamic force along the centerline for various voltages. It is evident that the electro-hydrodynamic force is negligible around the flame regardless of the magnitude of imposed voltages, reflecting the charge neutrality in this region. As the electric potential gradient develops between the flame and electrodes, an axial electro-hydrodynamic force is induced. For a given local electric field, the force acts in the same direction with the electric field for positive charges and in the opposite direction for negative charges. With the negative voltages imposed at the fuel side (cathode), the electric force in the space between the flame and cathode is opposed to the fuel stream because of the abundance of positive ions (see Fig. 6(a)). As negative ions dominate the charge density near the anode for negative voltages (see Fig. 6(c)), the electric force between the flame and anode points in the direction opposite to that of the velocity in the oxidizer stream. For the positive polarity condition, however, the magnitude of the electro-hydrodynamic force between the flame and anode is negligible, since the densities of negative charges are extremely low in this region as negative ions are hardly formed due to the absence of $\mathrm{O}_{2}$. As a result, the electro-hydrodynamic force is directed towards the cathode all the way. This explains the asymmetric behavior of the flame under two polarities of voltage, as observed in Figs. 2 and 3.

The new position of the flame is the result of the fluid-dynamic response to the electric forces, namely the ionic wind, induced in both upward and downward directions.

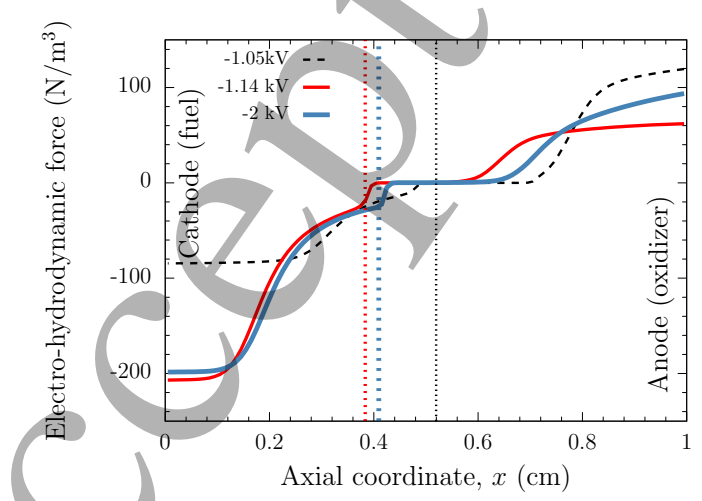

(a) Negative voltages

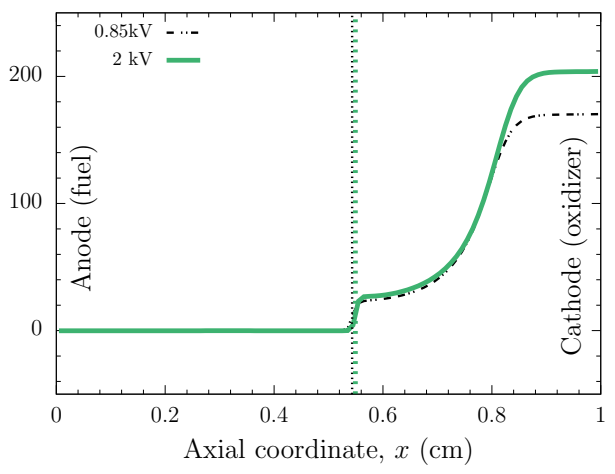

(b) Positive voltages

Figure 9. Distribution of axial electro-hydrodynamic force along the centerline $(r=0)$ for various voltages. Vertical dashed lines correspond to the flame locations, i.e. axial coordinate on the centerline where heat release rate peaks. 
Considering that the gas flow velocity and species molecular diffusion are small compared to the drift velocities $\mathbf{v}_{d, k}=\mu_{k} \mathbf{E}$, the total force $\mathbf{F}$ acting on the neutral molecules (see Eq. 5) is approximated from charged particle current densities and mobilities as:

$$
\mathbf{F} \approx \sum_{k}^{N} \frac{\mathbf{J}^{k}}{\mu^{k}},
$$

which can be written as:

$$
\mathbf{F} \approx \frac{\mathbf{J}^{+}}{\mu^{+}}+\frac{\mathbf{J}^{e}}{\mu^{e}}+\frac{\mathbf{J}^{-}}{\mu^{-}},
$$

where subscripts,$+ e$, and - denote positive ions, electrons and negative ions, respectively. Therefore, the electric force acting on a charged species is proportional to the species' current density and inversely proportional to the species' mobility.

With $\Delta V_{0}=-1.05 \mathrm{kV}$, the electric force opposed to the oxidizer stream is more significant than the one opposed to the fuel stream (towards the cathode) in the other side of the flame, which may explain the flame displacement towards the anode. The current density of negative ions is mainly controlled by the rate of formation of $\mathrm{O}_{2}^{-}$via the electron attachment to oxygen molecules and cannot theoretically exceed the current density of positive ions since $J_{\text {cathode }}^{+}=\left(J^{e}+J^{-}\right)_{\text {anode }}$ (conservation of charge). Therefore, the higher electric force towards the anode when $\Delta V_{0}=-1.05 \mathrm{kV}$ is due to a lower mobility of negative ions present near the anode $\left(\mathrm{O}_{2}^{-}, \mathrm{CHO}_{3}^{-}\right.$and $\mathrm{CO}_{3}^{-}$; see Fig. 7-a) compared to that of $\mathrm{H}_{3} \mathrm{O}^{+}$. Note that the mobility is inversely proportional to the molar mass of the species regardless of the location in the flame [30].

With $\Delta V_{0}=-2 \mathrm{kV}$, the electro-hydrodynamic force pointing towards the cathode is much larger than towards the anode, resulting in a flame relocation towards the cathode. The behavior is opposite for $\Delta V_{0}=-1.05 \mathrm{kV}$ case. By increasing the voltage, the current densities of positive ions and electrons increase, while the current density of negative ions decreases due to the considerable reduction of the number of electrons. As a result, the electric force beeomes more significant in the direction of positive ions:

$$
\left(\frac{J^{+}}{\mu^{+}}\right)_{\text {cathode }}>\left(\frac{J^{e}}{\mu^{e}}+\frac{J^{-}}{\mu^{-}}\right)_{\text {anode }} .
$$

Figure 9 may explain the unstable regime behavior (mentioned earlier in subsection 4.1) observed over a short interval of negative voltages in both experiments and simulations, where the flame does not reach a steady position (see movie in the Supplementary Material). Such a transient response can be explained by the lack of a balance between the flow momentum and the electric forces induced in both upward and downward directions. In this regime, the force controlling the direction of the flame movement change periodically due to modifications to the spatial distributions of temperature and reactive species caused by the flame movement, resulting in horizontal and vertical fluctuations of the flame. With positive voltages, the unstable regime was not observed since the force is always unidirectional such that the flame is pushed towards the cathode until a new momentum balance is reached. 


\subsection{Dynamics of the flow}

Figure 10 shows the streamlines obtained without an applied voltage and with a potential difference of $-3 \mathrm{kV}$. Without the electric field, a typical counterflow field with a stagnation plane at the center was obtained. The application of a voltage of $-3 \mathrm{kV}$ pushes the stagnation plane towards the electrode. The axial location of the stagnation plane in the reaction zone is correlated with that of the stoichiometric line $\left(Z=Z_{s t}=0.5\right)$. Similar behavior was found for other positive and negative voltages (not shown).

Profiles of the axial velocity along the centerline $(r=0)$ before and after the application of negative bias voltages are compared in Fig. 11. The general shape of the velocity profile is unchanged by the application of the electric field, but the flow field is shifted towards the cathode or the anode for a sufficiently high voltage. The same can be said for positive voltages (not shown). In summary, the electrohydrodynamic force reduces the local flow velocity in the region ahead of the reaction zone, and consequently the stagnation plane is adjusted to the new location, in the direction that can be identified from the electro-hydrodynamic force field shown in Fig. 9. As such, the stoichiometric mixture fraction location, which is an accurate measure of the nonpremixed flame position, also follows the behavior of the stagnation plane. Similar trends have also been reported in Ref. [59], where the displacement of a counterflow nonpremixed flame under AC electric field was found to be comparable with the displacement of the stagnation plane.

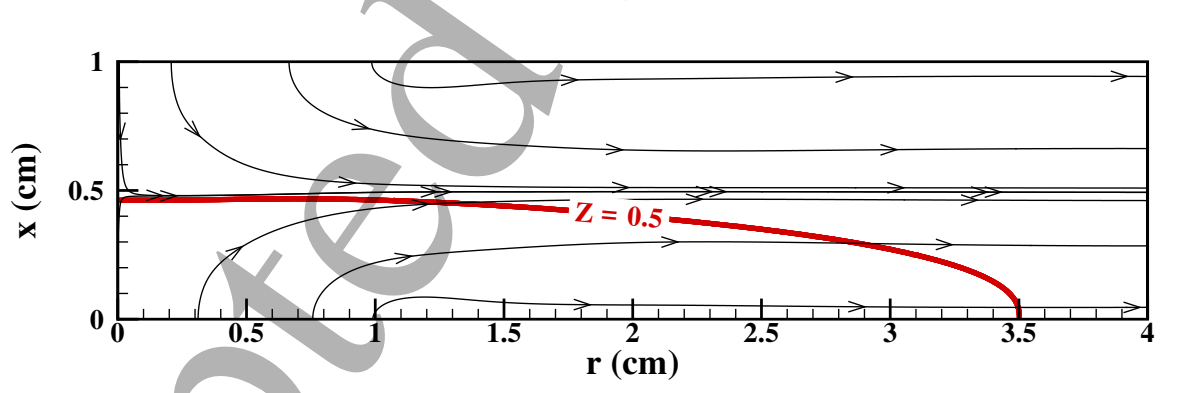

(a) $\Delta V_{0}=0 \mathrm{~V}$

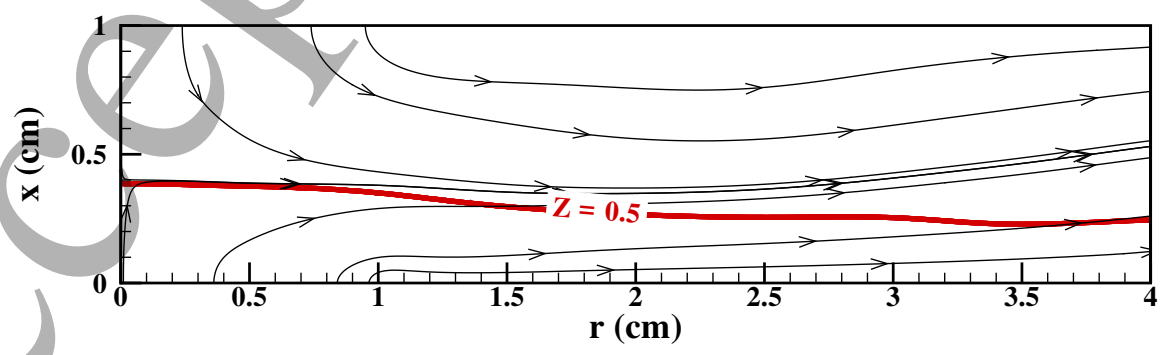

(b) $\Delta V_{0}=-3 \mathrm{kV}$

Figure 10. Streamlines with (a) no applied potential and (b) $\Delta V=-3 \mathrm{kV}$. Thick red line corresponds to $Z=Z_{s t}=0.5$. 


\section{Conclusions}

Figure 11. Axial velocity along the centerline $(r \triangleq 0)$ for various negative voltages. Vertical dashed lines correspond to the flame locations, i.e. axial coordinate on the centerline where heat release rate peaks.

A computational model to describe the electric field effect on methane/air flames was developed and two-dimensional numerical simulations for counterflow nonpremixed flames under DC bias voltages were undertaken. The main objective was to reproduce the flame behavior that is attributed to the electro-hydrodynamic effect, also known as the ionic wind effect, as observed in a recent experimental study. Incompressible fluid dynamic solver based on OpenFOAM was combined with a skeletal chemical kinetic mechanism and transport property evaluations developed previously by the authors' group.

The simulation reasonably reproduced the response of the flame position and current density to negative DC bias voltages at different intensity. Most notably, the simulation predicted the unstable regime at the voltage of $-1.1 \mathrm{kV}$, in good agreement with the experimental observation. Furthermore, the measured behavior of non-monotonic variation in the current density was also predicted with good qualitative agreement. The model provided good prediction of the flame position before the unstable regime and at saturation, but it showed important differences at the end of the unstable regime. Differences between simulations and experiments in the sub-saturation regime may be due to uncertainties in the prediction of transport coefficients of charged species.

The simulation further extended the parametric conditions at positive voltages, and the current-voltage behavior was found to be consistent with previous experimental results. The flame response to the electric field with positive and negative voltages exhibited qualitatively different behaviors. In the negative polarity, a non-monotonic variation of the current with the voltage was observed along with the existence of an unstable regime at an intermediate voltage level. With positive polarity, a typical monotonic current-voltage curve was obtained. This difference was attributed to the asymmetry in the distribution of the positive and negative charged species resulting 
from ionization processes.

Detailed examination of the spatial distributions of the charged species and electric field properties provided explanations of the observed and predicted anomaly such as the unstable regime and non-monotonic current-voltage characteristics in negative polarity. This behavior is attributed to a competition between two electric forces induced in both upward and downward directions. Further extensive studies are needed to understand the detailed mechanism of the instabilities caused by the electric field, as also observed in other experimental studies [56, 60]. Finally, the response of flow field to the applied electric field showed that the shift in the flame position was primarily attributed to the shift in the stagnation plane due to the electro-hydrodynamic forces.

The present study demonstrated that the employed models for the ion chemistry, transport, and fluid dynamics were able to describe the key processes responsible for the flame-electric field interaction. For quantitative prediction of the observed experimental behavior, accurate description of positive ions, negative ions, and electrons with distinct chemical and transport characteristics was found to be critical. Other detailed measurements obtained in different flame geometries $[15,56,59,60]$ may also be explored to assess the fidelity of the present models. These will be considered in future work.

\section{Acknowledgement}

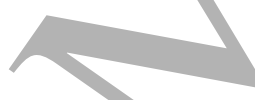

This research was funded by King Abdullah University of Science and Technology (KAUST) and made use of the computational resources managed by KAUST Supercomputing Lab (KSL).

\section{References}

[1] H.F. Calcote. Ion production and recombination in flames. Symp. (Int.) Combust., 8(1):184-199, 1961.

[2] J. Lawton and F. J. Weinberg. Electrical aspects of combustion. Oxford University Press, 1970.

[3] A. B. Fialkov. Investigations on ions in flames. Prog. Energy Combust. Sci., 23(5-6):399-528, 1997.

[4] R. I. Noorani and R. E. Holmes. Effects of electric fields on the blowoff limits of a methane-air flame. AIAA Journal, 23(9):1452-1454, 1985.

[5] S. M. Lee, C. S. Park, M.S. Cha, and S.H. Chung. Effect of electric fields on the liftoff of nompremixed turbulent jet flames. IEEE Trans. Plasma. Sci., 33(5):1703-1709, 2005.

[6] A. Ata, J. S. Cowart, A. Vranos, and B. M. Cetegen. Effects of direct current electric field on the blowoff characteristics of bluff-body stabilized conical premixed flames. Combust. Flame, 177(7):1291-1304, 2005.

[7] K. Criner, Cessou, J. Louiche, and P. Vervisch. Stabilization of turbulent lifted jet flames assisted by pulsed high voltage discharge. Combust. Flame, 144:422-425, 2006.

[8] M.K. Kim ans S.K. Ryu, S.H. Won, and S.H. Chung. Electric fields effect on liftoff and blowoff of nonpremixed laminar jet flames in a coflow. Combust. Flame, 157(1):17-24, 2010.

[9] M.K. Kim, S.H. Chung, and H.H. Kim. Effect of electric fields on the stabilization of premixed laminar bunsen flames at low ac frequency: Bi-ionic wind effect. Combust. Flame, 159(3):11511159, 2012. 
[10] M. Kono, F.B. Carleton, A.R. Jones, and F.J. Weinberg. The effect of non steady electric fields on sooting flames. Combust. Flame, 78(3-4):357-364, 1989.

[11] M. Saito, T. Arai, and M. Arai. Control of soot emitted from acetylene diffusion flames by applying an electric field. Combust. Flame, 119(3):356-366, 1999.

[12] Y. Wang, G.J. Nathan, Z.T. Alwahabi, K.D. King, K. Ho, and Q. Yao. Effect of a uniform electric field on soot in laminar premixed ethylene/air flames. Combust. Flame, 157(7):308-1315, 2010.

[13] H.C. Jaggers and A. von Engel. The effect of electric fields on the burning velocity of various flames. Combust. Flame, 16(3):275-285, 1971.

[14] R.J. Bowser and F.J. Weinberg. The effect of direct electric fields on normal burning velocity. Combust. Flame, 18(2):296-300, 1972.

[15] J. Kuhl, T. Seeger, L. Zigan, S. Will, and A. Leipertz. On the effect of ionic wind on structure and temperature of laminar premixed flames influenced by electric fields. Combust. Flame, 176:391-399, 2017.

[16] K. G. Payne and F. J. Weinberg. A preliminary investigation of field-induced ion movement in flame gases and its applications. Proc. Roy. Soc. A., 250:316-336, 1959.

[17] M. Belhi, P. Domingo, and P. Vervisch. Direct numerical simulation of the effect of an electric field on flame stability. Combust. Flame, 157(12):2286-2297, 2010.

[18] M. Belhi, P. Domingo, and P. Vervisch. Modeling of the effect of DC and AC electric fields on the stability of a lifted diffusion methane/air flame. Combust. Theor. Model., 17(4):749-787, 2013.

[19] D.G. Park, S.H. Chung, and M.S. Cha. Bidirectional ionic wind in nonpremixed counterflow flames with dc electric fields. Combust. Flame, 168:138-146, 2016.

[20] S.D. Marcum and B.N. Ganguly. Electric-field-induced flame speed modification. Combust. Flame, 143:27-36, 2005.

[21] D.L. Wisman, S.D. Marcum, and B.N. Ganguly. Electrical control of the thermodiffusive instability in premixed propane-air flames. Combust. Flame, 151:639-648, 2007.

[22] Dae Geun Park, SukHo Chung, and MinSuk Cha. Visualization of ionic wind in laminar jet flames. Combust. Flame, 184:246-248, 2017.

[23] J. Hu, B. Rivin, and E. Sher. The effect of an electric field on the shape of co-flowing and candle-type methane-air flames. Exp. Therm. Fluid Sci, 21:124-133, 2000.

[24] K. Yamashita, S. Karnani, and D. Dunn-Rankin. Numerical prediction of ion current from a small methane jet flame. Combust. Flame, 156(6):1227-1233, 2009.

[25] N. Speelman, M. Kiefer, D. Markus, U. Maas, L. P. H de Goey, and J. A. van Oijen. Validation of a novel numerical model for the electric currents in burner-stabilized methane air flames. Proc. Combust. Inst., 35(1):847-854, 2014.

[26] N. Speelman, L. P. H. de Goey, and J. A. van Oijen. Development of a numerical model for the electric current in burner stabilized methane-air flames. Combust. Theor. Model., 19(2):159-187, 2015.

[27] J. Han, M. Belhi, T.A. Casey, F. Bisetti, H.G. Im, and J.Y. Chen. The $i-V$ curve characteristics of burner-stabilized premixed flames: detailed and reduced models. Proc. Combust. Inst., $36(1): 1241-1250,2017$.

[28] M. Belhi, J. Han, T. A. Casey, J. Y. Chen, H. G. Im, S. M. Sarathy, and F. Bisetti. Analysis of the current-voltage curves and saturation currents in burner-stabilized premixed flames with detailed ion chemistry and transport models. Combust. Theor. Model., submitted(TCTM-2017-05-40), 2017.

[29] J. Prager, U. Riedel, and J. Warnatz. Modeling ion chemistry and charged species diffusion in lean methane-oxygen flames. Proc. Combust. Inst., 31(1):1129-1137, 2007.

[30] J. Han, M. Belhi, F. Bisetti, and S. M. Sarathy. Numerical modeling of ion transport in flames. Combust. Theor. Model., 19(6):744-772, 2015.

[31] F. Bisetti and M. El-Morsli. Calculation and analysis of the mobility and diffusion coefficient of thermal electrons in methane/air premixed flames. Combust. Flame, 159(12):3518-3521, 2012.

[32] J. F. Grcar F. M. Rupley A.E. Lutz, R. J. Kee. OPPDIF: A Fortran program for computing 
opposed-flow diffusion flames. Sandia National Laboratories, 1997.

[33] F. Bisetti and M. El Morsli. Kinetic parameters, collision rates, energy exchanges and transport coefficients of non-thermal electrons in premixed flames at sub-breakdown electric field strengths. Combust. Theor. Model., 18:148-184, 2014.

[34] J. han. Numerical Study of Electric Field Enhanced Combustion. PhD thesis, King Abdullah University of Science and Technology, 2017.

[35] T. Poinsot and D. Veynante. Theorical and Numerical Combustion. R.T. Edwards, 2005.

[36] J. Lawton and F. J. Weinberg. Maximum ion currents from flames and the maximum practical effects of applied electric fields. Proc. Roy. Soc. A (Lond), 277:468-497, 1963.

[37] J. P. Boeuf and L. C. Pitchford. Electrohydrodynamic force and aerodynamic flow acceleration in surface dielectric barrier discharge. J. Appl. Phys., 97(103307), 2005.

[38] V. Giovangigli. Multicomponent Flow Modeling. Birkhäuser, Boston, 1999.

[39] E.A. Mason and E.W. McDaniel. Transport Properties of Ions in Gases. John Wiley \& Sons, Inc., 1988.

[40] E.A. Mason, R.J. Munn, and J.F. Smith. Transport coefficients of ionized gases. Phys. Fluids, 10(8):1827-1832, 1967.

[41] M. Belhi, D. G. Park, J. Han, H. G. Im, M. S. Cha, and F. Bisetti. Numerical and experimental study of the current-voltage curves in atmospheric-pressure burner-stabilized premixed flames of c1-c4 fuels. Proceedings of the 8th European Combustion Meeting, pages 2293-2298, 2017.

[42] LXcat 2012. Phelps $\left(\mathrm{N}_{2}, \mathrm{CO}\right)$, Morgan $\left(\mathrm{O}_{2}, \mathrm{H}_{2}, \mathrm{CO}_{2}, \mathrm{H}_{2} \mathrm{O}\right)$, and Hayashi $\left(\mathrm{CH}_{4}\right)$ databases. http://www.lxcat.laplace.univ-tlse.fr, 2012.

[43] S.M. Burke, W. Metcalfe, O. Herbinet, F. Battin-Leclerc, F.M. Haas, J. Santner, F.L. Dryer, and H.J. Curran. An experimental and modeling study of propene oxidation. part 1: speciation measurements in jet-stirred and flow reactors. Combust. Flame, 161(11):2765-2784, 2014.

[44] S.M. Burke, U. Burke, R. Mc Donagh, O. Mathieu, I. Osorio, C. Keesee, A. Morones, E.L. Petersen, W. Wang, T.A. DeVerter, M.A. Oehlschlaeger, B. Rhodes, R.K. Hanson, D.F. Davidson, B.W. Weber, C.-J. Sung, J. Santner, Y. Ju, F.M. Haas, F.L. Dryer, E.N. Volkov, E.J.K. Nilsson, A.A. Konnov, M. Alrefae, F. Khaled, A. Farooq, P. Dirrenberger, P.A. Glaude, F. Battin-Leclerc, and H.J. Curran. An experimental and modeling study of propene oxidation. part 2: ignition delay time and flame speed measurements. Combust. Flame, 162(2):296-314, 2015.

[45] D. McElroy, C. Walsh, A. J. Markwick, M. A. Cordiner, K. Smith, and T. J. Millar. The UMIST database for astrochemistry 2012. A\&A, 550(A36):1-13, 2013.

[46] A. Burcat. Prof. Burcat's thermodynamic data: Ideal gas thermodynamic data in polynomial form for combustion and air pollution use. http://garfield.chem.elte.hu/Burcat/burcat.html, 2006.

[47] D. Kim, F. Rizzi, K. W. Cheng, J. Han, F. Bisetti, and O. M. Knio. Uncertainty quantification of ion chemistry in lean and stoichiometric homogenous mixtures of methane, oxygen, and argon. Combust. Flame, 162(7):2904-2915, 2015.

[48] S. Mahadevan and L.L. Raja. Simulations of direct-current air glow discharge at pressures $\sim 1$ torr: Discharge model validation. J. Appl. Phys., 107(093304), 2010.

[49] J.M. Goodings, D.K. Bohme, and C.W. Ng. Detailed ion chemistry in methane-oxygen flames. I. positive ions. Combust. Flame, 36:27-43, 1979.

[50] J.M. Goodings, D.K. Bohme, and C.W. Ng. Detailed ion chemistry in methane-oxygen flames. II. negative ions. Combust. Flame, 36:45-62, 1979.

[51] A. B. Alquaity, B. Chen, J. Han, H. Selim, M. Belhi, Y. Karakaya, T. Kasper, S. M. Sarathy, F. Bisetti, and A Farooq. New insights into methane-oxygen ion chemistry. Proc. Combust. Inst., 36(1):1213-1221, 2017.

[52] R.J. Kee, J.A. Miller, and G.H. Evans. A computational model of the structure and extinction of strained, opposed flow, premixed methane-air flames. Symp. (Int.) Combust., 22(2):479-1494, 1989.

[53] Openfoam foundation ltd. www.openfoam.org. Version: 2.4.0.

[54] J.C. Butcher. Numerical Methods for Ordinary Differential Equations. New York: John Wiley \& 
Sons, 2003.

[55] M.S. Cha and P.D. Ronney. Propagation rates of nonpremixed edge flames. Combust. Flame, 146(1-2):312-328, 2006.

[56] S. Karnani and D. Dunn-Rankin. Detailed characterization of dc electric field effects on small non-premixed flames. Combust. Flame, 162(7):2865-2872, 2015.

[57] A.N. Hayhurst, J.M. Goodings, and S.G. Taylor. The effects of applying electric fields on the mass spectrometric sampling of positive and negative ions from a flame at atmospheric pressure. Combust. Flame, 161(12):3249-3262, 2014.

[58] K.G. Xu. Plasma sheath behavior and ionic wind effect in electric field modified flames. Combust. Flame, 161(6):1678-1686, 2014.

[59] Carmen Guerra-Garcia and Manuel Martinez-Sanchez. Counterflow nonpremixed flame dc displacement under ac electric field. Combust. Flame, 162:4254-4263, 2015.

[60] Jesse Tinajero, Guillaume Bernard, Louise Autef, and Derek Dunn-Rankin. Characterizing i-v curves for non-premixed methane flames stabilized on different burner configurations. Combust. Sci. Technol., 189(10):1739-1750, 2017. 